УДК 621.01/.03

\title{
РАСЧЕТНО-ЭКСПЕРИМЕНТАЛЬНАЯ МЕТОДИКА ОЦЕНКИ ОСТАТОЧНОГО РЕСУРСА ГАЗОПРОВОДА ПО УСТАЛОСТНОЙ ПРОЧНОСТИ
}

\author{
Сызранцев Владимир Николаевич', \\ v_syzrantsev@mail.ru \\ Сызранцева Ксения Владимировна', \\ kv.syzr@gmail.com \\ 1 Тюменский индустриальный университет,
Россия, 625000, г. Тюмень, ул. Володарского, 38.
}

Актуальность. В реальных эксплуатационных условиях газопроводы работают при переменном уровне давления, которое в общем случае является величиной случайной, с неизвестным законом распределения. Также случайной является и температура окружающей среды. Вследствие отмеченных условий напряжения, возникающие в стенках трубы, не могут быть описаны законами, исследованными в рамках теории параметрической статистики. Основой прогнозирования ресурса газопровода является кривая усталости материала трубы, связывающая амплитуду действующих напряжений не со временем работы испытываемых до разрушения образцов, а с числом циклов их деформирования. Для определения в процессе эксплуатации газопровода остаточного ресурса во времени необходимо оценивать накопление в его материале усталостных повреждений при любом законе распределения напряжений независимо от его сложности. В настоящее время методики прогнозирования остаточного ресурса газопроводов, учитывающие фактический спектр изменения внешних нагрузок и процессы накопления от них усталостных повреждений в стенке трубы, отсутствуют. Поскольку газопроводы являются потенциально опасными объектами, определение их остаточного ресурса по времени эксплуатации является задачей важнейшей.

Цель: определение во временном диапазоне остаточного ресурса газопровода с заданной вероятностью неразрушения, подвергающегося в процессе эксплуатации воздействию случайного спектра внешних нагрузок независимо от сложности спектра, с учетом процессов накопления усталостных повреждений в газопроводе.

Методы: кинетическая теория механической усталости, методы непараметрической статистики, измерение циклических деформаций с помощью датчиков деформаций переменной чувствительности.

Результаты. Разработана расчетно-экспериментальная методика, основанная на комплексном использовании: кинетической теории механической усталости, учитывающей накопление повреждений в процессе циклического деформирования изделий; методов непараметрической статистики, обеспечивающих восстановление функции плотности распределения напряжений независимо от сложности закона их изменения в процессе эксплуатации газопровода; оригинальных средств измерения циклических деформаций - датчиков деформаций переменной чувствительности. Рассмотрены основные этапы реализации методики. По зафиксированной в процессе эксплуатации газопровода на датчике величине перемещения границы его реакции (первых «темных пятен») на основе разработанных в рамках кинетической теории усталости уравнений получены математические зависимости решения задачи определения эквивалентных по повреждающему воздействию чисел циклов нагружения для восстановления длительности ступеней блока напряжений, расчета эквивалентных по повреждающему воздействию напряжений. На примере реализации методики установлена зависимость остаточного ресурса газопровода в зависимости от величины реакции на датчике, позволяющая оперативно оценивать остаточный ресурс различных участков газопровода в условиях эксплуатации. Показан вариант использования результатов реализации методики для определения влияния коррозионного дефекта газопровода на его остаточный ресурс.

\section{Ключевые слова:}

Газопровод, датчики деформаций переменной чувствительности, теория механической усталости, эквивалентные числа циклов, эквивалентные напряжения, остаточный ресурс.

\section{Характеристика проблемы прогнозирования} остаточного ресурса газопроводов в эксплуатации

В реальных эксплуатационных условиях газопроводы работают при переменном уровне давления, которое в общем случае является величиной случайной с неизвестным законом распределения. Также случайной является и температура окружающей среды. Вследствие отмеченных условий напряжения, возникающие в стенках трубы, не могут быть описаны законами, исследованными в рамках теории параметрической статистики [1]. Помимо этого, и предельные напряжения, используемые при реализации методик определения прочности газопровода, также являются величинами случайными. Поскольку газопроводы являются потенциально опасными объектами, определение их надежности в процессе эксплуатации является задачей важнейшей.
Основными показателями, используемыми в настоящее время для определения надежности газопроводов [2], являются: безотказность, оцениваемая по вероятности безотказной работы, и долговечность - ресурс.

Традиционные методики расчета вероятности безотказной работы различных изделий $[3,4]$, в том числе и газопроводов [2, 5], как правило, базируются на описании случайных величин действующих и предельных напряжений нормальным законом распределения. Для учета фактических законов распределения напряжений в работе [1] предложены и реализованы методики расчета вероятности безотказной работы газопроводов, в которых неизвестные законы распределения действующих и предельных напряжений восстанавливаются на основе их выборок с использованием математиче- 
ского аппарата непараметрической статистики [6-10]. Данный подход распространен и на другую оценку безотказности газопровода - определение, независимо от сложности законов распределения действующих и предельных напряжений, квантилей коэффициента запаса прочности [1]. В данном подходе процессы накопления усталостных повреждений в материале трубы при эксплуатации газопровода не учитываются, что не позволяет научно обосновать значения вероятности безотказной работы, принимаемых в качестве предельных, соответствующих отказу газопровода.

Для организации планово-предупредительных профилактических и ремонтных работ газопровода более информативен другой показатель его надежности: долговечность, в вероятностной постановке - гамма $(\gamma)$ процентный ресурс по усталостной прочности $[2,4,5]$. Несмотря на почти двухсотлетние исследования, единственными достоверными данными об усталостной прочности материала являются те, которые получены в процессе циклического деформирования испытываемых образцов до их разрушения при нескольких уровнях действующих напряжений [4, 11-14]. Результаты испытаний образцов на долговечность представляются в виде кривой усталости (кривой Велера) [15-17], которая связывает напряжения $\left(\sigma_{a}\right)$ и числа циклов $(N)$ до разрушения образцов.

Выделим основные проблемы решения задачи прогнозирования $\gamma$-процентного ресурса газопровода на основе кривой усталости.

Анализ экспериментальных данных $\sigma_{a k}, \lg N_{k}$, $k=\overline{1, M}$ свидетельствует о близкой к прямолинейной их связи, в результате для описания кривой усталости традиционно используется линейная регрессионная зависимость, соответствующая $\gamma=50 \%$ вероятности разрушения образцов. Для прогнозирования $\gamma$-процентного ресурса необходима соответствующая допустимой вероятности раз-

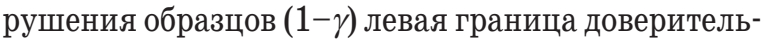
ного интервала, рассчитываемая на основе статистической обработки данных. Применение здесь метода линейного регрессионного анализа корректно лишь для распределений случайных величин $\sigma_{a}$ и $\lg N$ по нормальному закону $[3,4]$. Как показано в работе [18], вследствие логарифмирования оценки линейной модели кривой усталости смещаются, левые границы ее доверительных интервалов, рассчитанные с учетом восстановленных методами непараметрической статистики [1] фактических законов распределения $N$, приводят к ошибкам прогнозирования чисел циклов до поломки в десятки и более процентов.

Левая граница доверительного интервала кривой усталости при $\sigma_{a}=$ const позволяет рассчитать число циклов деформирования $N$, соответствующее вероятности неразрушения $\gamma$. В процессе эксплуатации напряжения в газопроводе изменяются по законам случайных величин при этом прогнозирование $\gamma$-процентного ресурса предполагает его оценку не в числе циклов, а во временном диапазоне. Из сказан- ного следует, что описанная выше модель обработки данных усталостных испытаний позволяет рассчитывать $\gamma$-процентный ресурс газопровода когда известны напряжения, эквивалентные случайному закону их изменения в газопроводе, и числа циклов, эквивалентные случайному процессу деформирования газопровода в эксплуатации. Поскольку при работе газопровода деградация его прочности связана с накоплением в материале усталостных повреждений, как эквивалентные напряжения, так и эквивалентные числа циклов нагружения необходимо определять исходя из их повреждающего воздействия. Решение этих задач в рамках линейных регрессионных моделей, описывающих кривые усталости, предполагающих за каждый цикл деформирования внесение величины поврежденности, равной $1 / N$, не представляется возможным.

Цель работы - определение во временном диапазоне остаточного ресурса газопровода с заданной вероятностью неразрушения, подвергающегося в процессе эксплуатации воздействию случайного спектра внешних нагрузок независимо от сложности спектра, с учетом процессов накопления усталостных повреждений в газопроводе, для достижения которой разработана расчетно-экспериментальная методика. Методика основана на результатах, полученных в трех различных направлениях научных исследований.

Первое направление связано с развитием кинетической теории механической усталости [17-19], в рамках которой для описания кривой малоцикловой усталости получено следующее уравнение:

$$
N\left(\sigma_{a}\right)=\left(1-10^{\frac{\sigma_{a}-\overline{\sigma_{b}}}{\vartheta}}\right) Q_{T} B_{0} \ln \left[1-\exp \left(A_{0} C_{0} \sigma_{a}\right)\right]
$$

где

$$
\begin{gathered}
B_{0}=\frac{\ln \left\{1+\left[\exp \left(\frac{\overline{\sigma_{b}}-\sigma_{R}}{\sigma_{R}-\sigma_{R T}}\right)-1\right]^{-1}\right\}}{\overline{\sigma_{b}}} ; \\
C_{0}=-\frac{\overline{\sigma_{b}}}{\left(\sigma_{R}-\sigma_{R T}\right)\left(\overline{\sigma_{b}}-\sigma_{R}\right)} ; A_{0}=\frac{D}{1-D},
\end{gathered}
$$

$D$ - степень усталостного повреждения $0 \leq D_{0} \leq D \leq D_{k} \leq 1 ; D_{0}$ - исходная поврежденность материала; $D_{k}$ - величина повреждения, соответствующая моменту действия последнего (разрушающего) цикла с напряжением $\sigma_{a} ; \vartheta$ - параметр, характеризующий наклон кривой усталости в системе координат $\lg N-\sigma_{a} ; Q_{T}-$ коэффициент, учитывающий сопротивление материала детали росту усталостных трещин; $\bar{\sigma}_{b}$ - математическое ожидание предела прочности материала; $\sigma_{R}$ - величина предела выносливости материала детали для условий ее циклического деформирования при коэффициенте асимметрии цикла $R ; \sigma_{R T}-$ предел текучести при циклическом деформировании.

В работе [18] на примере конкретных данных усталостных испытаний образцов $\sigma_{a k}, N_{k}, k=\overline{1, M}$ 
рассмотрены алгоритмы определения параметров модели (1), построение кривых усталости с различной величиной накопленных повреждений, расчета границ доверительных интервалов.

Второе направление исследований связано со статистической обработкой случайных величин, не описываемых законами, предложенными и исследованными в теории вероятности и математической статистике. Для определения любой сложности априори неизвестной функции плотности распределения (ФПР) случайной величины используется аппарат непараметрической статистики [1, 20-24], следуя которому ФПР $f_{n}(y)$ на основе выборки случайной величины $x_{i}, i=1, n$ описывается с помощью оценки Парзена-Розенблатта $[9,10]$ :

$$
f_{n}(y)=\frac{1}{n h_{n}} \sum_{i=1}^{n} K\left[\left(y-x_{i}\right) / h_{n}\right],
$$

где $K\left[\left(y-x_{i}\right) / h_{n}\right]$ - ядерная функция; $h_{n}$ - параметр размытости (ширина окна Парзена-Розенблатта).

Оптимальная величина $h_{n}^{*}$ определяется в результате поиска максимума функционала $[1,8]$ :

$$
\begin{gathered}
\max J\left(h_{n}^{*}\right)= \\
=\max _{h_{n}}\left\{\frac{1}{n} \sum_{i=1}^{n} \ln \left[\frac{1}{(n-1) h_{n}} \sum_{j \neq i}^{n-1} K\left(\frac{x_{i}-x_{j}}{h_{n}}\right)\right]\right\} .
\end{gathered}
$$

В рамках третьего направления разработаны оригинальные средства экспериментальной оценки напряжений при циклическом деформировании деталей - датчики деформаций интегрального типа (ДДИТ) [25]. Датчики требуемой конфигурации вырезают из электроосажденной фольги, подвергнутой специальной термомеханической обработке [25-28]. Датчики с помощью клея закрепляют в исследуемом месте детали. В процессе циклического деформирования шероховатость поверхности датчика изменяется. При прямом освещении поверхности изменение шероховатости - реакция датчика, воспринимается как «темные пятна». Момент возникновения первых «темных пятен» зависит от числа циклов деформирования и амплитуды циклического напряжения [25, 29]. В результате развития данного направления созданы датчики деформаций переменной чувствительности (ДДПЧ) [30, 31], изготавливаемые на основе ДДИТ, подвергнутых предварительной циклической наработке на образцах специальной формы в условиях известного их напряженно-деформированного состояния. После наработки ДДИТ до появления на их поверхности реакции в виде «темных пятен» датчики с образца снимают, они представляют собой ДДПЧ. Граница первых «темных пятен» на ДДПЧ отделяет на его поверхности рабочую зону, в пределах которой, несмотря на отсутствие на поверхности датчика реакции, его материал накапливает усталостные повреждения в соответствии с заданным на этапе наработки напряженно-деформированным состоянием образца. Вдоль рабочей части ДДПЧ величина накопленных повреждений является переменной, она зада- ется геометрической формой образца, на котором выполняется наработка датчика.

Преимуществами ДДПЧ являются [31]:

- отсутствие инкубационного периода до появления на датчике реакции;

- существенное упрощение процедуры регистрации реакции датчика, заключающееся в измерении по длине его рабочей части величины линейного перемещения $\left(x_{g}\right)$ границы первых «темных пятен»;

- возможность фиксации реакции на датчике в любой момент времени испытаний детали или узла.

Технология изготовления ДДПЧ, процедуры их тарирования, математическое описание тарировочных зависимостей, алгоритмы решения задач определения по реакции ДДПЧ напряжений, накопленных усталостных повреждений, эквивалентных напряжений при известных параметрах блока изменения напряжений подробно рассмотрены в работе [31].

\section{Основные этапы расчетно-экспериментальной методики прогнозирования $\gamma$-процентного остаточного ресурса газопровода}

Условия, при которых реализуется методика.

1. Для материала трубы имеется кривая усталости (рис. 1), параметры и границы доверительных интервалов которой рассчитаны на основе кинетической теории механической усталости [18] путем обработки данных $\sigma_{a k}, N_{k}, k=\overline{1, M}$ испытаний образцов до поломки, изготовленных из американской трубы HS-80.

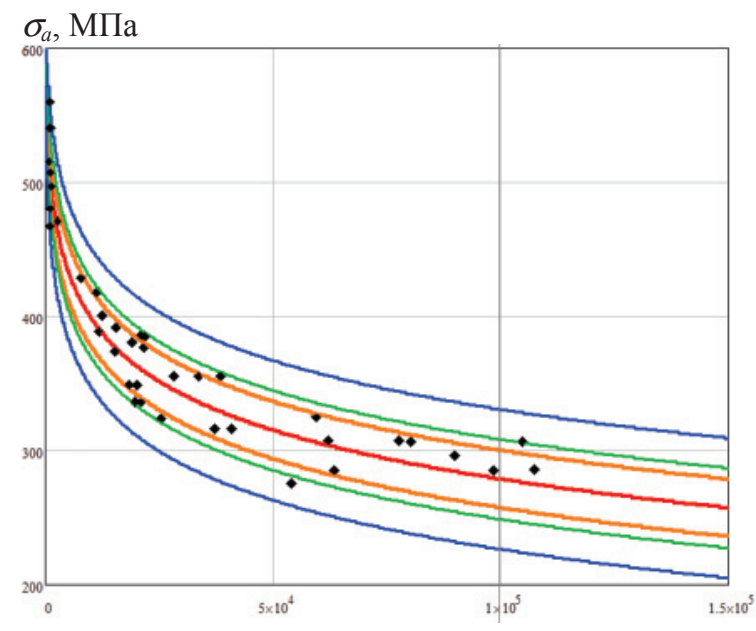

$N$

Puс. 1. Кривая усталости материала трубы (красная) с гранииами $90 \%$ (оранжевая), $95 \%$ (зеленая) и $99 \%$ (синяя) доверительных интервалов

Fig. 1. Fatigue curve (red) of pipe material with confidence limits $90 \%$ (orange), $95 \%$ (green) and $99 \%$ (blue)

Изложенная в работе [18] процедура обработки экспериментальных данных испытаний образцов на долговечность позволила для данных $\sigma_{a k}, N_{k}$, $k=\overline{1, M}$, отраженных на рис. 1 , получить следующие значения параметров, входящих в модель (1): 
$D=D_{0}=6,006 \cdot 10^{-11} ; \quad Q_{T}=1,53 \cdot 10^{6} ; \quad \bar{\sigma}_{b}=602,1 \mathrm{M \Pi a} ;$ $\vartheta=-121,811 ; \sigma_{R T}=201,914 \mathrm{M \Pi а} ; \sigma_{R}=263,621 \mathrm{M \Pi а}$.

2. Изготовлены ДДПЧ [30, 31], в пределах рабочей части которых определена зависимость (рис. 2) величины накопленных повреждений $D_{x}\left(x_{g}\right)$ [31] - тарировочная зависимость ДДПЧ.

3. В течение всего времени эксплуатации газопровод подвергается воздействию внешних нагрузок, процесс изменения которых является случайным и аддитивным.

4. Задано время $\left(T_{\text {н }}\right)$ работы газопровода до наклейки на него ДДПЧ. После чего, в течение времени $\left(T_{g}\right)$, газопровод эксплуатировался вместе с ДДПЧ. По истечении времени $T_{g}$ путем измерения величины смещения границы первых «темных пятен» $x_{g}$ зафиксирована реакция датчика на полученные усталостные повреждения.

Реализация методики позволяет во временном диапазоне осуществлять прогноз с заданной вероятностью не разрушения $(\gamma)$ остаточного ресурса $\left(T_{0}^{\gamma}\right.$, лет) газопровода по критерию усталостной прочности.

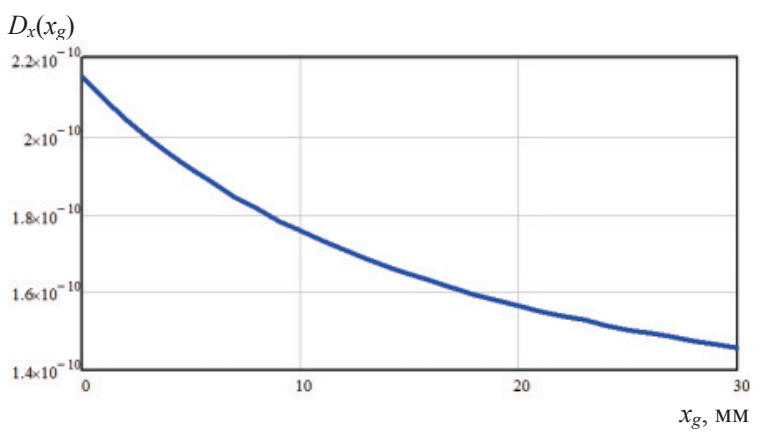

Puc. 2. Зависимость $D_{x}\left(x_{g}\right)$

Fig. 2. Dependence $D_{x}\left(x_{g}\right)$
Методология решения задачи представлена на рис. 3 и состоит из ряда последовательных этапов.

Эman 1 заключается в сборе информации о нагрузках, действующих на газопровод в течение отрезка времени из $T_{\text {н }}$, или периода $T_{g}$ (рис. 3 ), путем фиксирования (например, ежедневно, в течение года) значений давления $P_{i}, i=\overline{1, n=365}$ (рис. 4) и температуры $T_{i}, i=\overline{1, n=365}$ (рис. 5 ). Перед реализацией этого этапа на исследуемый участок газопровода помещают (наклеивают) ДДПЧ и после времени $T_{g}$ эксплуатации газопровода, измеряя смещение $x_{g}$ границы первых «темных пятен» по длине рабочей части ДДПЧ, фиксируют его реакцию на полученные усталостные повреждения.

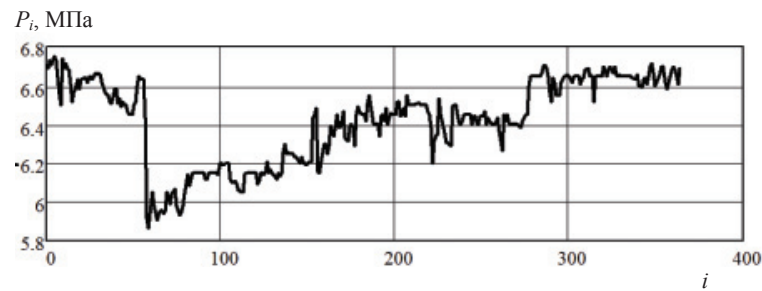

Pис. 4. Значения давлений $P_{i}($ МПа) в газопроводе

Fig. 4. Pressure values $P_{i}(M \Pi a)$ in pipeline $T_{i},{ }^{\circ} \mathrm{C}$

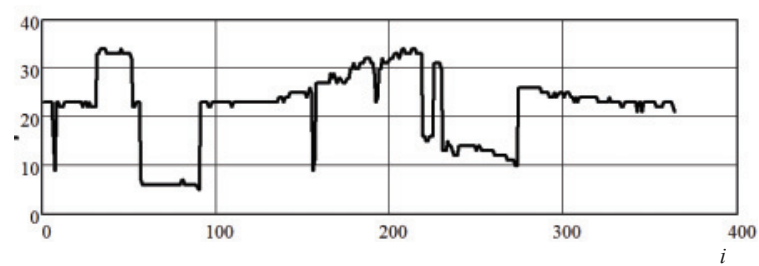

Puс. 5. Значения температуры $\left({ }^{\circ} \mathrm{C}\right)$

Fig. 5. Temperature values $\left({ }^{\circ} \mathrm{C}\right)$

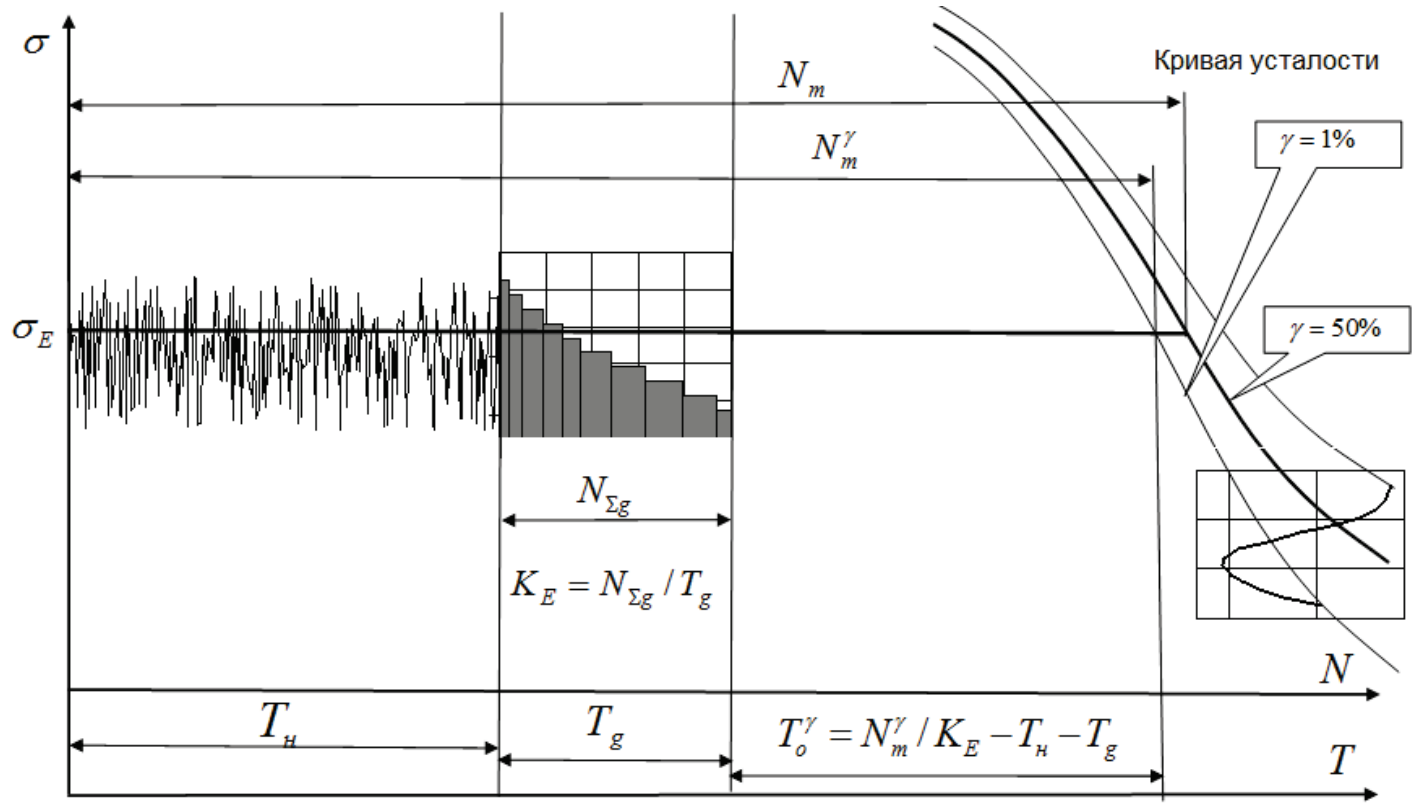

Рис. 3. Этапы реализации расчетно-экспериментальной методики

Fig. 3. Stages of implementation of computational and experimental method 

1, 2]:

Воспользовавшись известными выражениями

$$
\begin{gathered}
\sigma_{i}=\psi(h, l) \sqrt{\sigma_{\text {кц }, i}^{2}-\sigma_{\text {пр } j} \sigma_{\text {кц } i}+\sigma_{\text {пр } i}^{2}}, \\
\sigma_{\text {кц }, i}=\frac{P_{i} D_{\text {вн }}}{2 \delta} ; \\
\sigma_{\text {пр }, i}=-\alpha E T_{i}+\mu \sigma_{\text {кц }, i},
\end{gathered}
$$

где $\alpha=1,2 \cdot 10^{-5} ; \mu=0,3 ; E=2,15 \cdot 10^{5} \mathrm{M \Pi а}-$ коэффициенты Пуассона и линейного расширения материала, модуль его упругости; $\delta=20$ мм; $D_{\text {вн }}=1380 \mathrm{мм} \mathrm{-} \mathrm{толщина} \mathrm{стенки} \mathrm{и} \mathrm{внутренний} \mathrm{диа-}$ метр трубы; $\psi(h, l) \geq 1$ - коэффициент [1], отражающий увеличение напряжений вследствие наличия коррозионного дефекта глубиной $h$ и длиной $l$. Рассчитаем выборку возникающих в стенке трубы напряжений $\left(\sigma_{i}, i=\overline{1, n=365}\right)$, гистограмма которых отражена на (рис. 6).

По выборке $\left(\sigma_{i}, i=\overline{1, n}\right)$ методами непараметрической статистики [1], принимая в качестве ядерной функции нормальное ядро $[1,8]$, решая задачу (2), (3), восстановим необходимую для дальнейших расчетов ФПР напряжений $f_{n}(\sigma)$ :

$$
f_{n}(\sigma)=\frac{1}{n h_{\sigma}} \sum_{i=1}^{n} \frac{1}{\sqrt{2 \pi}} \exp \left[-0,5\left(\frac{\sigma-\sigma_{i}}{h_{\sigma}}\right)^{2}\right] \text {. }
$$

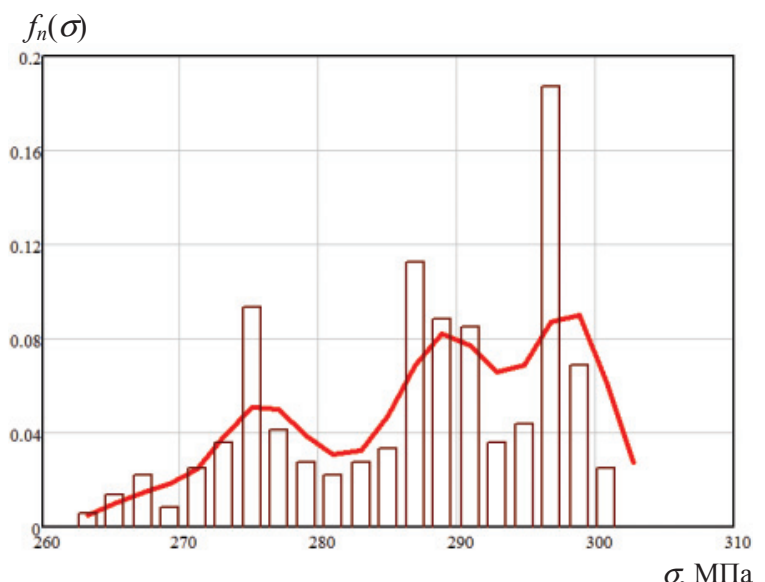

Puс.6. Гистограмма действующих напряжений в газопроводе и восстановленная функция $f_{n}(\sigma)$

Fig. 6. Histogram of stresses occurring in pipe and restored function $f_{n}(\sigma)$

Начальное значение параметра размытости $h_{\sigma}=2,879$ для задачи (3) рассчитывается по зависимости [1]:

$$
h_{\sigma}=D_{\sigma} n^{-0,2}, \text { где } D_{\sigma}=\sqrt{\frac{1}{n-2} \sum_{i=1}^{n}\left(\sigma_{i}-\frac{1}{n} \sum_{i=1}^{n} \sigma_{i}\right)^{2}} .
$$

Оптимальная величина $h_{\sigma}(6)$, соответствующая максимуму функционала (3), равна $h_{\sigma}=2,368$, функция $f_{n}(\sigma)$, восстановленная методами непараметрической статистики, показана на рис. 6 линией.

На основе функции $f_{n}(\sigma)$ режим нагружения можно представить в виде ступенчатой циклограм- мы, содержащей конечное число $(m)$ ступеней блоков напряжений $\left(\sigma_{i}, j=\overline{1, m}\right)$. Сумма относительных продолжительностей ступеней $\left(t_{j}, j=\overline{1, m}\right)$, как и интеграл от функции $f_{n}(\sigma)$, равна единице. Для построения ступенчатой циклограммы нагружения диапазон изменения напряжений $\left(\sigma_{\min }=\min \left(\sigma_{i}\right)\right.$, $\sigma_{\max }=\max \left(\sigma_{i}\right)$ разбивается на $m$ интервалов (в рассматриваемом примере $m=10)$, ширина которых:

$$
h_{m}=\left(\sigma_{\max }-\sigma_{\min }\right) / m \text {. }
$$

В каждом $j$-м интервале рассчитывается его середина

$$
\sigma_{j}=\sigma_{\min }+h_{m} \frac{j}{2}+h_{m}(j-1)
$$

и на основе зависимости ФПР (5) для полученных значений $\sigma_{j}, j=\overline{1, m}$ вычисляются величины:

$$
f_{\sigma j}=f_{n}\left(\sigma_{j}\right) \text {. }
$$

Описанная процедура позволяет для каждой $j$-й ступени блока напряжений $\sigma_{j}, j=1, m$ рассчитать значение относительной продолжительности $j$-й ступени по формуле:

$$
t_{j}=f_{\sigma j} \frac{\sigma_{j}}{\sum_{j=1}^{m} f_{\sigma j} \sigma_{j}} .
$$

Располагая ступени блока $\sigma_{j}, t_{j}, j=\overline{1, m}$, вычисленные в соответствии с формулами (7) и (8), в порядке снижения уровня напряжений, получаем необходимую для решения задачи гистограмму изменения напряжений в газопроводе. Для рассматриваемого закона (рис. 6) блок изменения напряжений показан на рис. 7.

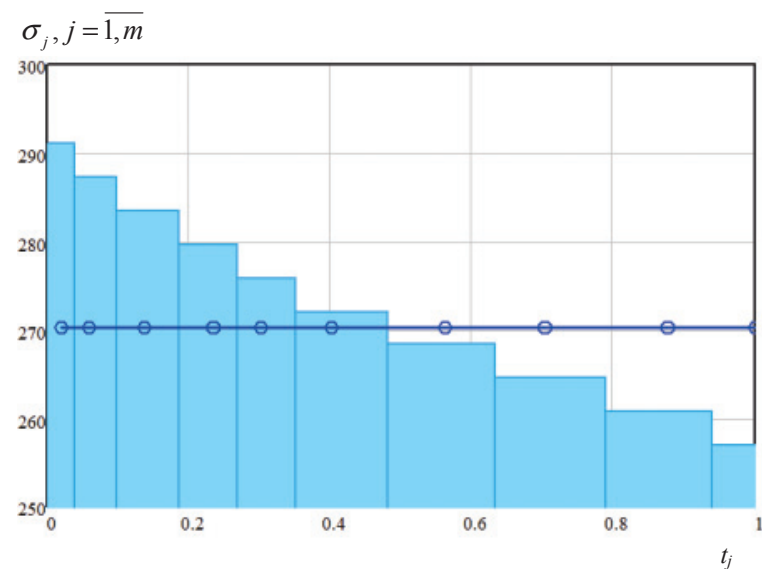

Puc. 7. Блок изменения напряжений для ФПР $f_{n}(\sigma)$ рис. 6

Fig. 7. Block of stress change for distribution density function $f_{n}(\sigma)$ showed in Fig. 6

Эman 2. При зафиксированной после работы трубопровода с ДДПЧ величине $x_{g}$ совместно с установленным блоком изменения напряжений (рис. 7) определяется эквивалентное по повреждающему воздействию число циклов $N_{\Sigma g}$ деформирования газопровода за время его эксплуатации с ДДПЧ. 
Методика решения этой задачи основана на использовании уравнения (1) в соответствии с алгоритмом, рассмотренным в работе [31].

Воспользуемся тарировочной зависимостью для ДДПЧ (рис. 2), отражающей изменение поврежденности $D_{x}\left(x_{g}\right)$ датчика в пределах его рабочей части. Наработка ДДПЧ выполняется на образце с конической рабочей частью (угол конуса $\alpha$ ) в условиях изгиба с вращением образца при величине изгибающего момента $(M)$ в течение $N_{g}$ числа циклов деформирования до появления на датчике реакции в виде первых «темных пятен» в сечении образца диаметром $d_{g}$. Действующее в этом сечении напряжение $\left(\sigma_{g x}\right)$, соответствующее значению параметра $x_{g}=0$, известно:

$$
\sigma_{g x}=\frac{32 M}{\left[\pi\left(2 \operatorname{tg} \alpha x_{g}+d_{g}\right)\right]^{3}} .
$$

Поврежденность ДДПЧ $D_{g 0}=D_{x}\left(x_{g}=0\right)$ на границе первых «темных пятен» на основе уравнения (1) рассчитывается с учетом (9) при $x_{g}=0$ по выражению:

$$
D_{g 0}=D_{0}+\frac{E_{g}}{E_{g}+C_{0}},
$$

где

$$
\begin{gathered}
E_{g}=\left(\sigma_{g x}\right)^{-1} \ln \left\{1-\exp \left[\frac{N_{g n}}{P_{0} Q_{T} B_{0}}\right]\right\}, \\
P_{0}=1-10^{\left(\sigma_{g x}-\overline{\sigma_{b}}\right) / 9}, \\
N_{g n}=P_{0} Q_{T} B_{0} \ln \left[1-\exp \left(A_{0} \sigma_{g x}\right)\right]-N_{g}, \\
A_{0}=C_{0} \frac{D_{0}}{1-D_{0}} .
\end{gathered}
$$

Расчет поврежденности датчика $D_{x}\left(x_{g}\right)$ в сечении, задаваемом координатой $x_{g}=$ const, выполняется по зависимости (10) при значении $\sigma_{g x}$, вычисленном по формуле (9).

Если в процессе эксплуатации газопровода с ДДПЧ его реакция - граница первых «темных пятен», сместилась на величину $x_{g}=$ const, то датчик получил дополнительное повреждение, равное:

$$
D_{g t}=D_{g 0}-D_{x}\left(x_{g}\right) .
$$

Это же повреждение получил материал трубы газопровода за пока неизвестное суммарное число циклов деформирования $N_{\Sigma g}$ в условиях изменения напряжений в соответствии с установленным выше (рис. 6) блоком нагружения.

Фиксируя величину $N_{\Sigma g}=$ const, определим длительности ступеней блока нагружения:

$$
N_{j}=N_{\Sigma g} t_{j}, j=\overline{1, m} .
$$

Значения $N_{i}$, полученные по формуле (12), с учетом известных величин $\sigma_{i}, j=1, m$, позволяют рассчитать повреждения материала трубы $D_{i}\left(N_{\Sigma g}\right)$, $j=\overline{1, m}$ после реализации каждой $j$-й ступени блока нагружения и определить суммарную величину повреждения:

$$
D_{\Sigma}\left(N_{\Sigma g}\right)=\sum_{j=1}^{m} D_{j}\left(N_{\Sigma g}\right) .
$$

Расчет $D_{j}\left(N_{\Sigma g}\right), j=\overline{1, m}$ включает первоначальное обнуление значений $D_{j}\left(N_{\Sigma g}\right)=0$ и дальнейшие последовательные вычисления для каждого $j=\overline{1, m}$ по формулам, полученным на основе уравнения (1):

$$
\begin{gathered}
A_{j}\left(N_{\Sigma g}\right)=\frac{D_{0}+\sum_{k=1}^{j} D_{k}\left(N_{\Sigma g}\right)}{1-\left(D_{0}+\sum_{k=1}^{j} D_{k}\left(N_{\Sigma g}\right)\right)}, \\
P_{j}=1-10^{\left(\sigma_{j}-\overline{\sigma_{b}}\right) / \vartheta},
\end{gathered}
$$

$$
\begin{aligned}
& N_{n j}\left(N_{\Sigma g}\right)=P_{j} Q_{T} B_{0} \ln \left\{1-\exp \left[A_{j}\left(N_{\Sigma g}\right) C_{0} \sigma_{j}\right]\right\} ; \\
& E_{j}\left(N_{\Sigma g}\right)=\sigma_{j}^{-1} \ln \left\{1-\exp \left[\frac{N_{n j}\left(N_{\Sigma g}\right)-N_{j}}{P_{j} Q_{T} B_{0}}\right]\right\} ; \\
& D_{j}\left(N_{\Sigma g}\right)=\frac{E_{j}\left(N_{\Sigma g}\right)}{E_{j}\left(N_{\Sigma g}\right)+C_{0}}-\left[D_{0}+\sum_{k=1}^{j} D_{k}\left(N_{\Sigma g}\right)\right] .
\end{aligned}
$$

Поскольку рассчитанная по формуле (13) с учетом (14) на основе кинетической теории усталости величина $D_{\Sigma}\left(N_{\Sigma g}\right)$ должна быть равна зафиксированному с помощью ДДПЧ значению $D_{g i}$, имеем следующее, трансцендентное относительно $N_{\Sigma g}$, уравнение:

$$
D_{\Sigma}\left(N_{\Sigma g}\right)-D_{g t}=0 .
$$

Установленное в результате решения уравнения (11) число циклов $N_{\Sigma g}$ полностью восстанавливает параметры $\sigma_{i}, N_{j}=N_{\Sigma g} \cdot t_{j}, j=\overline{1, m}$ всех $m$ ступеней блока изменения напряжений.

Эman 3. Определение напряжения $\sigma_{E}$, эквивалентного по повреждающему воздействию блоку напряжений $\sigma_{j}, N_{j}, j=\overline{1, m}$, восстановленному в результате реализации второго этапа методики. Для решения задачи воспользуемся работой [31].

За все $m$ ступеней блока материал трубопровода получит повреждение (13) $D_{\Sigma}\left(N_{\Sigma g}\right)$, с учетом которого выражение для кривой усталости (1) запишем в виде:

$$
N_{D}\left(\sigma_{a}\right)=\left(1-10^{\frac{\sigma_{a}-\overline{\sigma_{b}}}{\vartheta}}\right) Q_{T} B_{0} \ln \left[1-\exp \left(A_{\Sigma} C_{0} \sigma_{a}\right)\right],
$$

где

$$
A_{\Sigma}=\frac{D_{\Sigma}\left(N_{\Sigma g}\right)+D_{0}}{1-D_{\Sigma}\left(N_{\Sigma g}\right)-D_{0}} .
$$

Для определения числа циклов до разрушения газопровода, деформирование которого происходит при амплитуде напряжений $\sigma_{a}=\sigma_{E}$, воспользуемся выражением (1) при $D=D_{0}$ :

$$
\begin{gathered}
N\left(\sigma_{E}\right)=\left(1-10^{\frac{\sigma_{E}-\overline{\sigma_{b}}}{\vartheta}}\right) Q_{T} B_{0} \ln \left[1-\exp \left(A_{0} C_{0} \sigma_{E}\right)\right], \\
A_{0}=D_{0} /\left(1-D_{0}\right) .
\end{gathered}
$$


Число циклов $N\left(\sigma_{E}\right)(17)$ по отношению к числу циклов, рассчитанному по зависимости (16) при $\sigma_{a}=\sigma_{E}$, отличается на величину $N_{\Sigma g}$. В результате имеем следующее уравнение:

$$
N_{D}\left(\sigma_{E}\right)+N_{\Sigma g}-N\left(\sigma_{E}\right)=0 .
$$

Уравнение (18) относительно $\sigma_{E}$ является трансцендентным, его решение выполняется численным методом.

Эman 4. Определенные в результате реализации третьего этапа рассматриваемой методики эквивалентное напряжение $\sigma_{E}$ и эквивалентное по повреждающему воздействию число циклов нагружения $N_{\Sigma g}$ газопровода за период его эксплуатации в течение времени $T_{g}$ являются исходными данными для прогнозирования остаточного ресурса газопровода на основе известной кривой усталости материала трубы (рис. 1). Решение задачи выполняется в следующей последовательности.

Рассчитывают коэффициент $K_{E}=N_{\Sigma g} / T_{g}$, имеющий размерность цикл/год при работе газопровода с ДДПЧ в течение года, который позволяет привести случайный режим эксплуатации газопровода к эквивалентному ему блочному режиму с установленными параметрами. Подставляя полученную величину $\sigma_{E}$ в описывающее кривую усталости математическое выражение (1) при $D=D_{0}$, рассчитывают медианное значение $N_{m}$ числа циклов до отказа трубы вследствие усталости, а используя левую границу доверительного интервала кривой усталости, соответствующую вероятности разрушения $1-\gamma$, определяют число циклов $N_{m}^{\gamma}$ с вероятностью неразрушения $\gamma$. Остаточный гамма процентный ресурс газопровода $T_{0}^{\gamma}$ (в годах) рассчитывают по зависимости:

$$
T_{\mathrm{o}}^{\gamma}=N_{m}^{\gamma} / K_{E}-T_{\mathrm{H}}-T_{g} .
$$

\section{Результаты расчета $\gamma$-процентного остаточного ресурса газопровода}

Реализацию второго, третьего и четвертого этапов разработанной расчетно-экспериментальной методики проиллюстрируем на примерах прогнозирования остаточного ресурса газопровода при различных величинах реакции $x_{g}$ ДДПЧ, зафиксированных за время эксплуатации газопровода с датчиком.

Кривая усталости материала трубы представлена на рис. 1. Параметры модели (1) кривой усталости: $\bar{\sigma}_{b}=602,1 \mathrm{MПа;} Q_{T}=1,53 \cdot 10^{6} ; \sigma_{R}=263,621 \mathrm{MПа;}$ $\sigma_{R T}=201,914 \mathrm{M \Pi а} ; D=D_{0}=6,006 \cdot 10^{-11} ; \vartheta=-121,811$. Коэффициенты Пуассона, линейного расширения материала и модуль его упругости имеют значения: $\alpha=1,2 \cdot 10^{-5} ; \mu=0,3 ; E=2,15 \cdot 10^{5}$ МПа. Толщина стенки и внутренний диаметр трубы соответственно равны: $\delta=20$ мм и $D_{\text {вн }}=1380$ мм. Гистограмма действующих в газопроводе напряжений показана на рис. 6. На рис. 7 эта гистограмма преобразована в совокупность десяти ступеней блока нагружения. Газопровод эксплуатировался в течение десяти лет $\left(T_{\mathrm{H}}=10\right)$ после чего в течение года $\left(T_{g}=1\right)$ с
ДДПЧ, тарировочная зависимость которого отражена на рис. 2.

На основе представленных данных осуществим решение задачи прогнозирования остаточного гамма-процентного $(\gamma=97,5 \%)$ ресурса газопровода при различных величинах реакции ДДПЧ $\left(x_{g}\right)$. Peзультаты решения трансцендентного уравнения (15) относительно эквивалентного числа циклов $\left(N_{\Sigma g}\right)$ нагружения газопровода отражены в таблице. При этом установленная в результате решения уравнения (18) величина эквивалентного напряжения $\sigma_{E}=270,300$ МПа.

\section{Таблица. Результаты расчетов \\ Table. Calculation results}

\begin{tabular}{|c|c|c|c|c|c|c|}
\hline $\begin{array}{c}x_{g}, \text { мм } \\
(\mathrm{mm})\end{array}$ & 1 & 5 & 10 & 15 & 20 & 25 \\
\hline$N_{\Sigma g}$ & 494,244 & $1,848 \cdot 10^{3}$ & $2,813 \cdot 10^{3}$ & $3,402 \cdot 10^{3}$ & $3,795 \cdot 10^{3}$ & $4,072 \cdot 10^{3}$ \\
\hline $\begin{array}{c}T_{0}^{\gamma} \text {, годы } \\
\text { (уеаг) }\end{array}$ & 123,832 & 25,061 & 12,69 & 8,589 & 6,56 & 5,365 \\
\hline $\begin{array}{c}T_{0}^{\gamma}, \text { годы } \\
\text { (years) }\end{array}$ & 102,012 & 19,299 & 8,962 & 5,534 & 3,839 & 2,841 \\
\hline
\end{tabular}

В предпоследней строке таблицы показаны рассчитанные по выражению (19) значения гаммапроцентного остаточного ресурса $\left(T_{0}^{\gamma}\right)$ газопровода, не имеющего коррозионные дефекты, по усталостной прочности. В качестве иллюстрации, на рис. 8 синей линией показана построенная на основе данных таблицы зависимость $T_{0}^{\gamma}\left(x_{g}\right)$.

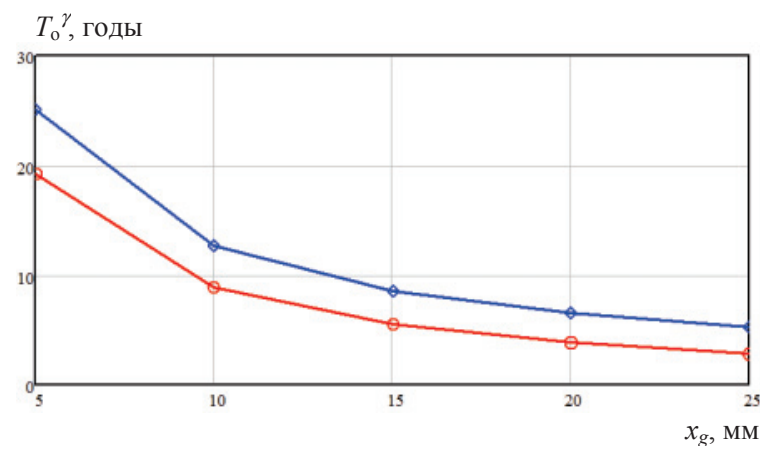

Pис. 8. Зависилость $T_{0}^{\gamma}\left(x_{g}\right)$

Fig. 8. Dependence $T_{0}^{\gamma}\left(x_{g}\right)$

В том случае, когда газопровод имеет коррозионный дефект глубиной $h=2 \mathrm{mм}$ и длиной $l=200 \mathrm{Mм}$, коэффициент концентрации $\varphi(h, l)$, входящий в формулу (4), на основании зависимостей работы [32], имеет значение $\varphi(h, l)=1,035$. Выполненные по вышеизложенной методике расчеты величины $T_{0}^{\gamma}\left(x_{g}\right)$ для этого случая при вариации $x_{g}$ представлены в последней строке таблицы и на рис. 8 полученная функция $T_{0}^{\gamma}\left(x_{g}\right)$ показана красной линией. Определенная в результате решения уравнения (18) величина эквивалентного напряжения $\sigma_{E}=279,771$ МПа. Из анализа рис. 8 следует, что наличие коррозионного дефекта глубиной $h=2 \mathrm{мm}$ и длиной $l=200 \mathrm{mм}$ уменьшает в $1,5 \ldots 1,7$ раза остаточный гамма-процентный 
$(\gamma=97,5 \%)$ ресурс газопровода. Это снижение остаточного ресурса соответствует всем коррозионным дефектам глубиной $h$ и длиной $l$, величина которых соответствует решению уравнения $\varphi(h, l)=1,035$.

\section{Заключение}

Для решения задачи оценки остаточного ресурса газопровода по усталостной прочности разработана методика, основанная на комплексном использовании кинетической теории механической усталости, учитывающей накопление повреждений в процессе циклического деформирования изделий, методов непараметрической статистики, обеспечивающих восстановление функции плотности распределения напряжений независимо от сложности закона их изменения в процессе эксплуатации газопровода, оригинальных средств измерения циклических деформаций - датчиков деформаций переменной чувствительности. Рассмотрены основные этапы реализации метода. На основе зафиксированной в процессе эксплуатации газопровода величины перемещения границы реакции (первых «темных пятен») на датчике и разработан-

\section{СПИСОК ЛИТЕРАТУРЫ}

1. Оценка безопасности и прочностной надежности магистральных трубопроводов методами непараметрической статистики / В.Н. Сызранцев, В.В. Новоселов, П.М. Созонов, С.Л. Голофаст. - Новосибирск: Наука, 2013. - 218 с.

2. Махутов Н.А., Пермяков В.Н. Ресурс безопасной эксплуатации сосудов и трубопроводов. - Новосибирск: Наука, 2005. $516 \mathrm{c.}$

3. Kapur K.C., Lamberson L.R. Reliability in Engineering Design. New York: John Wiley \& Sons, 1977. - 586 p.

4. Collins J.A. Failure of Materials in Mechanical Design: Analysis, Prediction, Prevention. - New York: John Wiley \& Sons, 1981. $630 \mathrm{p}$.

5. Целостность и безопасность трубопроводных систем / С.А. Тимашев, А.В. Бушинская, М.Г. Малюкова, Л.В. Полуян. - Екатеринбург: Уральское отделение Российской Академии Наук, 2013. $-590 \mathrm{c}$.

6. Vapnik V. An overview of statistical learning theory // IEEE Transactions on Neural Networks. - 1999. - V. 10. - Iss. 5. P. 988-999.

7. Орлов А.И. Прикладная статистика. - М.: Экзамен, 2004. $656 \mathrm{c.}$

8. Simakhin V.A. Nonparametric robust regression estimate // Proceedings SPIE. - 2006. - V. 6522. - P. 130-139.

9. Parzen E. On estimation of a probability density function and mode // Annals of Mathematical Statistics. - 1962. - V. 33. - № 3. P. $1065-1076$

10. Rozenblatt M. Remarks on some nonparametric estimates of a density functions // Annals of Mathematical Statistics. - 1956. V. 27. - № 3. - P. 832-837.

11. Когаев В.П., Гадолина И.В. Расчет деталей машин при нерегулярном режиме нагружения // Машиноведение. - 1991. № 5. - C. $45-50$.

12. Fowler S., Toumpis A., Galloway A. Fatigue and bending behaviour of friction stir welded DH36 steel // International Journal of Advanced Manufacturing Technology. - 2016. - V. 84. - Iss. 9. P. 2659-2669. DOI: $10.1007 / \mathrm{s} 00170-015-7879-3$.

13. Когаев В.П., Дроздов Ю.Н. Прочность и износостойкость деталей машин. - М.: Высшая школа, 1991. - 319 с. ных в рамках кинетической теории усталости уравнений получены математические зависимости для решения задачи определения эквивалентных по повреждающему воздействию чисел циклов нагружения для восстановления длительности ступеней блока напряжений, расчета эквивалентных по повреждающему воздействию напряжений.

На примере обработки экспериментальных данных по величине возникающих при работе газопровода давлений и температуры рассмотрена процедура получения зависимости его остаточного гамма-процентного ресурса по усталостной прочности от зафиксированной на датчике реакции. Данная зависимость позволяет оперативно количественно оценивать остаточный ресурс различных участков газопровода в условиях эксплуатации. Представлен вариант расчета остаточного ресурса газопровода при наличии в нем коррозионного дефекта. Установлено, что дефекты глубиной $h$ и длиной $l$, при которых вызываемый дефектами коэффициент концентрации напряжений соответствуют значению $\varphi(h, l)=1,035$, снижают остаточный ресурс газопровода в $1,5 . . .1,7$ раза.

14. Даль В. Поведение стали при циклических нагрузках. - M: Meталлургия, $1982 .-568$ с.

15. Susmel L., Lazzarin P. A bi-parametric Wöhler curve for high cycle multiaxial fatigue assessment // Fatigue \& Fracture of Engineering Materials \& Structures. - 2002. - V. 25 . - P. 63-78. D0I: $10.1046 / j .1460-2695.2002 .00462 . x$

16. Schijve J. Fatigue of structures and materials in the $20^{\text {th }}$ century and the state of the art // International Journal of Fatigue. 2003. - V. 25. - P. 679-702.

17. Почтенный Е.К. Прогнозирование долговечности и диагностика усталости машин. - Минск: Наука и техника, 1983. - 246 с.

18. Сызранцев В.Н., Сызранцева К.В. Обработка данных многоцикловых испытаний на основе кинетической теории усталости и методов непараметрической статистики: монография. - Тюмень: Изд-во Тюменского государственного нефтегазового университета, 2015. - 135 с.

19. Почтенный Е.К. Оценка циклической прочности деталей машин // Вестник машиностроения. - 1969. - № 9. - С. 11-15.

20. Sonawane M.S., Dhawale C.A. Evaluation and analysis of few parametric and nonparametric classification methods // Proc. $2^{\text {nd }}$ International Conference on Computational Intelligence and Communication Technology (CICT 2016). - Ghaziabad, India, 2016. - Article number 7546567. - P. 14-21. DOI: 10.1109/CICT.2016.

21. Liu Y., Zhang J., Zhao X. A new nonparametric screening method for ultrahigh-dimensional survival data // Computational Statistics and Data Analysis. - 2018. - V. 119. - P. 74-85.

22. A nonparametric test for slowly-varying non stationarities / D. Baptista de Souza, J. Chanussot, A-C. Favre, P. Borgnat // Signal Processing. - 2018. - V. 143. - P. 241-252.

23. Lindsey J.K. Introduction to Applied Statistics: a Modelling Approach. $2^{\text {nd }}$ ed. - Oxford, UK: Oxford University Press, 2004. $336 \mathrm{p}$.

24. Maronna R., Martin D., Yohai V. Robust Statistics: Theory and Methods. - NY: John Wiley and Sons, 2006. - 198 p.

25. Сызранцев В.Н., Голофаст С.Л., Сызранцева К.В. Диагностика нагруженности и ресурса деталей трансмиссий и несущих систем машин по показаниям датчиков деформаций интегрального типа. - Новосибирск: Наука, 2004. - 188 с. 
26. Голофаст С.Л. Диагностика работоспособности передач Новикова датчиками деформаций интегрального типа / под ред. В.Н. Сызранцева. - Новосибирск: Наука, 2004. - 164 с.

27. Investigation of various criteria for evaluation of aluminum thin foil «smart sensors» images / S.V. Panin, A.V. Eremin, P.S. Lyubutin, M.V. Burkov // IOP Conference Series: Material Science and Engineering. - 2014. - V. 66. - Article Number 012024.

28. Investigation of sensitivity of aluminum foil based strain sensors at fatigue damage evaluation of CFRP / M.V. Burkov, S.V. Panin, P.S. Lyubutin, A.V. Eremin // Adv. Mater. Res. - 2014. V. 1040. - P. 943-948.

29. Syzrantsev V., Syzrantseva K. The stress-strain condition estimation of detail in crack tip by integral strain gauges // IOP Conference Series: Material Science and Engineering - 2016. V. 127. - Article Number 012051.
30. Способ изготовления датчиков для контроля циклических деформаций: пат. Рос. Федерация № 2209412; заявл. 11.03.2001, опубл. 27.07.2003. - Бюл. № 21. - 4 с.

31. Сызранцев В.Н., Сызранцева К.В. Определение напряжений и остаточного ресурса по показаниям датчика деформаций интегрального типа переменной чувствительности // Известия Томского политехнического университета. Инжиниринг георесурсов. - 2017. - Т. 328. - № 9. - С. 82-93.

32. Оценка прочностного ресурса газопроводных труб с коррозионными повреждениями / И.Н. Бирилло, А.Я. Яковлев, Ю.А. Теплинский, И.Ю. Быков, В.Н. Воронин / под общ. ред. д-ра техн. наук, проф. И.Ю. Быкова. - М.: ЦентрЛитНефтеГаз, 2008. -168 c.

Поступила: 11.12.2018 2.

\section{Информация об авторах}

Cызранцев В.Н., доктор технических наук, профессор, Заслуженный деятель науки РФ, заведующий кафедрой машин и оборудования нефтяной и газовой промышленности Тюменского индустриального университета.

Cызранцева K.B., доктор технических наук, доцент кафедры кибернетических систем Тюменского индустриального университета. 


\title{
COMPUTATIONAL AND EXPERIMENTAL METHOD FOR EVALUATING GAS PIPELINE RESIDUAL SERVICE LIFE BY FATIGUE STRENGTH
}

\author{
Vladimir N. Syzrantsev', \\ v_syzrantsev@mail.ru \\ Ksenia V. Syzrantseva', \\ kv.syzr@gmail.com \\ Tyumen Industrial University,
38, Volodarsky street, Tyumen, 625000, Russia.
}

The relevance. Under operating conditions gas pipelines work at a time variable pressure level (in general, at a random level with unknown distribution law) and change (random variation relative to the annual trend) of ambient temperature. Due to the mentioned conditions, the stresses occurring in pipe walls are not described by the laws proposed and studied in the framework of the theory of parametric statistics. The basis for predicting a gas pipeline life is the pipe material fatigue curve connecting the amplitude of the actual stresses with the number of cycles of the tested sample deformations rather but not with the time of their operation until they are destroyed. In order to determine a gas pipeline residual service life in operation in the course of time, it is necessary to estimate a fatigue damage accumulation in its material under any law of stress distribution, regardless of its complexity. Currently, there are no methods for predicting gas pipeline residual service life, taking into account the actual range of changes in external loads and the processes of fatigue damage accumulation in pipe walls. Since gas pipelines are potentially dangerous objects, the determination of their residual life by the operation time is the most important task.

The main aim of the study is to determine gas pipeline residual service life in a time span with the given non-destruction probability, when the pipeline is exposed to a random range of external loads during its operation, regardless of the range complexity, taking into account the processes of fatigue damage accumulation in the pipeline

Methods: the kinetic theory of mechanical fatigue, methods of nonparametric statistics, measurement of cyclic deformations using variable sensitivity integral strain gauges.

Results. The authors have developed a computational and experimental method based on integrated use of the kinetic theory of mechanical fatigue, taking into account damage accumulation in cyclic deformation of products, methods of nonparametric statistics, providing restoration of function of stress distribution density regardless of the complexity of the law of their changes during pipeline operation and original means of measuring cyclic deformations - variable sensitivity integral strain gauges. The main stages of the method implementation was considered. The authors obtained the mathematical relations for determining the number of loading cycles, equivalent by damaging effect, to restore the duration of the stages of the stresses aggregate and to calculate the stresses, equivalent by damaging effect, according to the recorded by the gauge value of displacement of its response limits (the first "dark spots») during the gas pipeline operation based on the equations developed in the framework of the kinetic theory of fatigue. On the example of the method implementation, a relationship of gas pipeline residual life defined by the gauge response was established, which allows estimating quickly the residual service life of various sections of a gas pipeline under operating conditions. The paper demonstrates an alternative use of the method results to determine the effect of different amounts of gas pipeline corrosion defects on its residual service life.

Key words:

Gas pipeline, variable sensitivity integral strain gauges, mechanical fatigue theory,

equivalent numbers of cycles, equivalent stresses, residual service life.

\section{REFERENCES}

1. Syzrantsev V.N., Novoselov V.V., Sozonov P.M., Golofast S.L. Otsenka bezopasnosti i prochnostnoy nadezhnosti magistralnykh truboprovodov metodami neparametricheskoy statistiki [Assessment of safety and strength reliability of the transfer pipelines by methods of nonparametric statistics]. Novosibirsk, Nauka Publ., 2013. $218 \mathrm{p}$.

2. Makhutov N.A., Permyakov V.N. Resurs bezopasnoy ekspluatatii sosudov i truboprovodov [Resource of safe operation of vessels and pipelines]. Novosibirsk, Nauka Publ., 2005. 516 p.

3. Kapur K.C., Lamberson L.R. Reliability in Engineering Design. New York, John Wiley \& Sons, 1977. 586 p.

4. Collins J.A. Failure of Materials in Mechanical Design: Analysis, Prediction, Prevention. New York, John Wiley \& Sons, 1981. $630 \mathrm{p}$.

5. Timashev S.A., Bushinskaya A.V., Malukova M.G., Poluyan L.V. Tselostnost $i$ bezopasnost truboprovodnykh sistem [Integrity and safety of pipeline systems]. Ekaterinburg, Ural branch of the Russian Academy of Sciences Publ., 2013. 519 p.

6. Vapnik V. An overview of statistical learning theory. IEEE Transactions on Neural Networks, 1999, vol. 10, Iss. 5, pp. 988-999.
7. Orlov A.I. Prikladnaya statistika [Applied statistics]. Moscow, Examen Publ., 2006. 671 p.

8. Simakhin V.A. Nonparametric robust regression estimate. Proceedings SPIE, 2006, vol. 6522, pp. 130-139.

9. Parzen E. On estimation of a probability density function and mode. Annals of Mathematical Statistics, 1962, vol. 33, no. 3, pp. $1065-1076$.

10. Rozenblatt M. Remarks on some nonparametric estimates of a density functions. Annals of Mathematical Statistics, 1956, vol. 27, no. 3, pp. 832-837.

11. Kogaev V.P., Gadolina I.V. Analysis of machine parts in unsteadystate loading conditions. Mashinovedenie, 1991, Iss. 5, pp. 45-50.

12. Fowler S., Toumpis A., Galloway A. Fatigue and bending behaviour of friction stir welded DH36 steel. International Journal of Advanced Manufacturing Technology, 2016, vol. 84, Iss. 9, pp. 2659-2669. D0I: 10.1007/s00170-015-7879-3.

13. Kogaev V.P., Drozdov Y.N. Prochnost i iznosostoykost detaley mashin [Strength and durability of machine parts]. Moscow, Vysshaya shkola Publ., 1991. 319 p.

14. Dal V. Povedenie stali pri tsiklicheskikh nagruzkakh [Steel behavior under cyclic loads]. Moscow, Metallurgiya Publ., 1983. $568 \mathrm{p}$. 
15. Susmel L., Lazzarin P. A bi-parametric Wöhler curve for high cycle multiaxial fatigue assessment. Fatigue \& Fracture of Engineering Materials \& Structures, 2002, vol. 25, pp. 63-78. DOI: $10.1046 / \mathrm{j} .1460-2695.2002 .00462 . \mathrm{x}$

16. Schijve J. Fatigue of structures and materials in the $20^{\text {th }}$ century and the state of the art. International Journal of Fatigue, 2003, vol. 25, pp. 679-702.

17. Pochtenny E.K. Prognozirovanie dolgovechnosti $i$ diagnostika ustalosti mashin [Longevity forecasting and fatigue diagnostics of machines]. Minsk, Nauka i tekhnika Publ., 1983. 246 p.

18. Syzratnsev V.N., Syzrantseva K.V. Obrabotka dannykh mnogotsiklovykh ispytaniy na osnove kineticheskoy teorii ustalosti i metodov neparametricheskoy statistiki [Data processing of high-cycle tests on the basis of the kinetic theory of fatigue and methods of nonparametric statistics]. Tyumen, Tyumen State Oil and Gas University Publ., 2015. $135 \mathrm{p}$.

19. Pochtenny E.K. Otsenka tsiklicheskoy prochnosti detaley mashin [Estimation of cyclic durability of machine parts]. Russian Engi neering Research, 1969, no. 9, pp. 11-15.

20. Sonawane M.S., Dhawale C.A. Evaluation and analysis of few parametric and nonparametric classification methods. Proc. $2^{\text {nd }}$ In ternational Conference on Computational Intelligence and Communication Technology (CICT 2016). Ghaziabad, India, 2016. Article number 7546567. pp. 14-21. DOI: 10.1109/CICT.2016.

21. Liu Y., Zhang J., Zhao X. A new nonparametric screening method for ultrahigh-dimensional survival data. Computational Statistics and Data Analysis, 2018, vol. 119, pp. 74-85.

22. Baptista de Souza D., Chanussot J., Favre A-C., Borgnat P. A nonparametric test for slowly-varying non stationarities. Signal Processing, 2018, vol. 143, pp. 241-252.

23. Lindsey J.K. Introduction to Applied Statistics: a Modelling Approach. $2^{\text {nd }}$ ed. Oxford, UK, Oxford University Press, 2004. $336 \mathrm{p}$.

24. Maronna R., Martin D., Yohai V. Robust Statistics: Theory and Methods. NY, John Wiley and Sons, 2006. 198 p.

25. Syzrantsev V.N., Golofast S.L., Syzrantseva K.V. Diagnostika nagruzhennosti $i$ resursa detaley transmissiy $i$ nesushchikh si- stem mashin po pokazaniyam datchikov deformatsiy integralnogo tipa [Diagnostics of loading and resource of parts of machines transmissions and carrying systems]. Novosibirsk, Nauka Publ., $2004.188 \mathrm{p}$.

26. Golofast S.L. Diagnostika rabotosposobnosti peredach Novikova datchikami deformatsiy integralnogo tipa [Serviceability diagnostics of Wildhaber-Novikov gearings by integral strain gauges]. Novosibirsk, Nauka Publ., 2004. 164 p.

27. Panin S.V., Eremin A.V., Lyubutin P.S., Burkov M.V. Investigation of various criteria for evaluation of aluminum thin foil «smart sensors» images. IOP Conference Series: Material Science and Engineering, 2014, vol. 66, Article Number 012024.

28. Burkov M.V., Panin S.V., Lyubutin P.S., Eremin A.V. Investigation of Sensitivity of Aluminum Foil Based Strain Sensors at Fatigue Damage Evaluation of CFRP. Adv. Mater. Res, 2014, vol. 1040 , pp. 943-948.

29. Syzrantsev V., Syzrantseva K. The stress-strain condition estimation of detail in crack tip by integral strain gauges. IOP Conference Series: Material Science and Engineering, 2016, vol. 127, Article Number 012051.

30. Syzrantsev V.N. Sposob izgotovleniya datchikov dlya kontrolya tsiklicheskikh deformatsiy [Method for manufacturing sensors for monitoring cyclic deformations]. Patent RF, no. 2209412, 2003.

31. Syzrantsev V., Syzrantseva K. Determination of stresses and residual life in accordance with indications of variable-sensitivity integral strain gauge. Bulletin of the Tomsk Polytechnic University, Geo Assets Engineering, 2017, vol. 328, Iss. 9, pp. 82-93. In Rus.

32. Birillo I.N., Yakovlev A.Ya., Teplinskiy Yu.A., Bykov I.Yu., Voronin V.N. Otsenka prochnostnogo resursa gasoprovodnykh trub $s$ korrozionnymi pourezhdeniyami [Assessment of strength resource of gas pipelines with corrosive damages]. Ed. by Prof. I.Yu. Bykov. Moscow, CentrLitNefteGaz Publ., 2008. 168 p.

Received: 11 December 2018.

\section{Information about the authors}

Vladimir N. Syzrantsev, Dr. Sc., full professor, Honorary worker of science of the Russian Federation, head of the department «Machines and equipment of oil and gas industry», Tyumen Industrial University.

Ksenia V. Syzrantseva, Dr. Sc., associate professor, Tyumen Industrial University. 